\section{LA APERTURA DEL ESPACIO DE TRABAJO ${ }^{1}$}

ALEJANDRO PRIETO
Alejandro Prieto / Arquitecto y Magíster en Arquitectura, Pontificia Universidad Católica de Chile, 2010. Fue coordinador del proyecto FONDECYT N¹1100143, Escuela de Arquitectura, UC. Actualmente es profesor de la Facultad de Arquitectura, Diseño y Estudios Urbanos de la UC.

"La vida es oficina, pero la oficina no es vida. El adulto occidental pasa la mitad de su existencia consciente en entornos administrativos, y la calidad ambiental de la mayor parte de ellos es difícilmente compatible con el estímulo: si la iniciativa se adormece en el espacio homogéneo y reglamentado, los sentidos se embotan bajo la luz sin sombras de un cielo artificial” (Fernández-Galiano, 2003)

La calidad ambiental del espacio de trabajo ha sido un tema de creciente interés desde fines del s. xx. A la incorporación paulatina de criterios de eficiencia energética y sostenibilidad en el desarrollo de edificios de oficina, se suma la validación de teorías de la psicología ambiental, que relacionan la percepción de confort del trabajador con su eficiencia y rendimiento en el desempeño de las actividades laborales.

Diversos estudios coinciden en que un punto clave en el desarrollo de ambientes de trabajo confortables es la relación entre el espacio interior y el medio ambiente externo, lo que permite defender una mayor apertura al ambiente natural, por su contribución a la generación de "lugares de trabajo sostenibles y saludables" (Loftness, 2008).

La revalorización de una relación directa con el medio ambiente en el espacio de trabajo, implica un cambio de paradigma importante respecto de la manera en que se ha desarrollado la tipología de edificios de oficina desde mediados del s. xx. Si bien sus inicios se remontan a fines del s. XIX, su consolidación se produjo gracias a los adelantos técnicos y la consiguiente mecanización del ambiente interior, que fue masificada después de la Segunda Guerra Mundial. La utilización de sistemas activos de control ambiental, como la iluminación artificial y el aire acondicionado, propició una separación total del entorno, incluso en el caso de edificios con fachada de muro cortina donde el interior constituye un entorno artificial separado ambientalmente del exterior (FIG.01). Así, al considerar el lugar de trabajo como un ambiente controlado artificialmente, sin relación con los agentes externos, surgió la posibilidad teórica de concebir espacios con las mismas características ambientales en toda su superficie, independientemente de la orientación y ubicación del edificio. La planta libre, entendida como superficie neutra dentro de un área simuladamente uniforme, fue planteada como elemento característico del edificio de oficinas moderno, debido a su amplia aceptación por parte de los desarrolladores inmobiliarios y empresarios de la época. En ese marco la flexibilidad en la organización de los puestos de trabajo, asociada al espacio neutro, se perfiló como la principal ventaja del modelo en su desarrollo inicial.

A partir de la revalorización de una apertura directa al medio ambiente, el concepto de flexibilidad en el espacio de trabajo entró en crisis. La incorporación de variables ambientales externas al interior desarma los esquemas establecidos, pues ya no se trata de un espacio neutro, sino de un ambiente claramente diferenciado en relación a su vínculo con el medio externo.

De la superficie total del espacio de trabajo, sólo un porcentaje puede aprovechar las ventajas de una apertura al ambiente exterior. De esta manera, se plantea una distinción entre el sector que no tiene esta posibilidad y el vinculado directamente a este. Entenderemos este último como superficie autónoma, al poder responder a los requerimientos de confort y aprovechar la influencia de agentes ambientales externos.
La superficie interior requerirá un fuerte apoyo mecánico para generar las condiciones ambientales que permitan su adecuada ocupación.

Los agentes ambientales externos inciden en el interior de diversas maneras, al interactuar con los requerimientos de confort de los trabajadores. Es evidente la importancia psicológica de contar con vistas hacia el exterior o la posibilidad de controlar el ingreso de aire fresco, sin embargo sus beneficios se extienden a un ahorro energético importante en la operación del espacio de trabajo por concepto de climatización. Esto no implica necesariamente una transparencia desmedida del sistema de fachada, sino que, por el contrario, lograr una integración adecuada del medio externo y aprovechar sus características propias para responder a las necesidades del espacio interior.

No obstante, la posibilidad de establecer relaciones con el exterior se restringe a la zona definida como superficie autónoma, por lo que no es muy arriesgado inferir que los edificios que cuentan con un mayor porcentaje de estos espacios, presentan también más potencial para generar espacios interiores confortables, además de una mayor factibilidad para ser climatizados en forma pasiva.

El nivel de superficie autónoma para edificios de oficina varía principalmente en función del ancho de la planta. Los edificios de crujías menores tendrán mayor porcentaje de superficie de trabajo expuesta directamente al medio ambiente exterior (FIG.02). Los primeros edificios de oficina, a fines del s. xIx y principios del xx, buscaban abrirse al ambiente exterior, para aprovechar las ventajas de la iluminación natural, dado que la luz de gas no era eficaz para sus requerimientos lumínicos. (Ábalos, 2000) Esto generó que las dimensiones de las crujías fueran normadas en función de la entrada de luz natural al espacio de trabajo. Precisamente esta intención inicial de aprovechar las ventajas de una apertura directa al exterior es lo que permite hablar actualmente de una revalorización de la integración del medio ambiente, perdida durante el apogeo de la climatización activa a mediados del s. xx.

Hoy cabe preguntar ¿es posible optimizar las superficies de trabajo de mayores dimensiones de manera de contar con mayor porcentaje de superficie autónoma y, de esa forma, mejorar el potencial de climatización pasiva para edificios de oficina?

Actualmente, se observa el desarrollo de soluciones innovadoras que intentan resolver este problema en edificios de crujías mayores y fomentan una mayor integración del entorno a partir de la utilización de espacios de transición. Estos se definen como espacios ubicados entre el ambiente exterior e interior, y actúa, al mismo tiempo, como zonas de amortiguación.

ESPACIOS DE TRANSICIÓN

La necesidad de relacionar el espacio de trabajo con el medio ambiente exterior, ha impulsando la búsqueda de una imagen que rompa con

1 Este artículo se basa en la investigación para la tesis Interfaz ambiental en edificios de oficina: envolvente de espesor programático variable como sistema de mediación ambiental pasiva, en proceso de desarrollo, bajo la tutoría del profesor Claudio Vásquez, en el programa de Magíster en Arquitectura de la Escuela de Arquitectura de la Pontificia Universidad Católica de Chile. 

la concepción hermética del edificio de oficinas, presente desde la segunda mitad de s. xx. Estos avances se presentan principalmente en Europa, debido al temprano rechazo hacia el modelo de planta libre y la existencia de regulaciones que buscan mejorar los niveles de confort ambiental de los trabajadores. De esta forma, el acceso directo a una ventana ha llegado a ser obligatorio en gran parte del territorio europeo.

Si bien existen proyectos en los que se ha optado por una reducción del ancho de la planta, para mejorar la relación entre cada recinto y el medio ambiente; cada vez se plantean más edificios de oficina que integran espacios de transición como estrategia de mediación. Estos se traducen principalmente en atrios y patios que consideran varios pisos de altura.

La razón principal para la utilización de espacios de transición es la necesidad de integrar el medio ambiente exterior a zonas que de otra forma no tendrían relación con este. Su incorporación beneficia la calidad del ambiente interior al aumentar el porcentaje de superficie autónoma, es decir, permite el ingreso de luz natural y aire fresco a zonas alejadas del perímetro y posibilita un menor consumo energético, al mismo tiempo que se propone una mejora en los niveles de confort.

Por otro lado, al relacionar las oficinas a través de estos espacios se plantea una apertura indirecta hacia el exterior, distinta al caso de oficinas situadas en el perímetro. Esto, lejos de ser un obstáculo, comprende un potencial especial para la generación de una adecuada mediación con el entorno, ya que permite una permeabilidad diferenciada frente al clima. Así, al contar con una zona de amortiguación climática es posible controlar las condiciones ambientales propias del contexto.

Un temprano ejemplo -que se ha convertido en un paradigma del uso de este tipo de espacios- es la sede central del Commerzbank en Frankfurt, Alemania, diseñada por Norman Foster en 1998 (FIG.03). El edificio se ubica en un predio triangular situado en una esquina, al costado de la anterior sede del banco. La intención inicial de ocupar todo el predio, comprendía la generación de plantas de grandes dimensiones, en las que la zona central quedaría incomunicada al ambiente exterior. Para resolver esto, se incorporó un atrio central de toda la altura del edificio, alrededor del cual se disponen las oficinas. Además, se diseñaron patios de cuatro pisos de altura que relacionan el atrio con el exterior y permiten el acceso de iluminación natural y aire fresco a las zonas interiores de la planta. De esta manera, no solo se utilizan espacios de transición para satisfacer los requerimientos de confort de los recintos contiguos, sino que se relacionan entre sí para generar un sistema de mediación con el contexto que mejora el desempeño ambiental del edificio completo (FIGS.04 y 05).

La manera en que se vinculan estos espacios constituye el partido general del edificio, lo que potencia un diseño íntimamente ligado a la necesidad de mediar con el entorno, como base para las operaciones posteriores. Se disponen núcleos de circulación en los vértices de la planta triangular, que relacionan los patios y permiten una mayor apertura de estos al minimizar su acción como elemento de obstrucción de luz y radiación solar (FIG.06).

La creciente utilización de estos espacios es potenciada por ciertos cambios en las actividades laborales, relacionados al intercambio de información y a la necesidad de impulsar la comunicación entre los trabajadores. De esta forma, "la oficina en altura extruida verticalmente, en que la comunicación entre pisos está restringida al ascensor y al estacionamiento, está siendo reevaluada. En lugar de esto, la creación de centros sociales y centros de servicio ha surgido, alrededor de escaleras abiertas y atrios" (Hartkopf, 1993).

A su vez, la incorporación de espacios exteriores es referida por algunos autores como un componente clave en la configuración de la oficina creativa. Su uso "permite que la oficina continúe más allá de los límites de la planta arquitectónica tradicional. Estos espacios exteriores han sido adoptados por compañías creativas, cuyos arquitectos han concebido inteligentemente el medio ambiente exterior para ser parte del espacio de trabajo" (Myerson, 1999).

La aparente validación del uso de espacios de transición en edificios de oficina ha generado, durante los primeros años del presente siglo, un incipiente desarrollo de soluciones que los contemplan y se enfocan en las ventajas que supone su utilización dentro de un sistema de mediación ambiental. En este sentido, la función de la envolvente como elemento mediador entre interior y exterior, adquiere mayor complejidad, ya que según la definición de Ábalos y Herreros, es "el espesor del cerramiento el que crece hasta generar los atrios con los que las oficinas inician su implicación urbana”. (Ábalos, 2000).

Así, el uso de espacios de transición se plantea como una evolución del sistema de envolvente. Esto reviste de gran importancia al diseño de la fachada, la que no puede ser entendida como un componente anexado durante el desarrollo del proyecto, sino que como parte fundamental de su concepción, bajo un concepto global de desempeño ambiental para el edificio. Concebir un mayor espesor para el cerra- miento, implica su división en capas sucesivas y separadas por una distancia variable que permita llevar a cabo distintas actividades. A partir del desarrollo de dobles pieles vidriadas hacia fines del s. xx y principios del xxI, o el uso de protecciones solares, se han observado sistemas de envolvente que contemplan múltiples capas, con un mayor espesor. Solo recientemente se ha comenzado a explorar la posibilidad de variar el espesor e incorporar espacios de transición como sistema de mediación.

Un ejemplo medianamente reciente es la embajada de Renania del Norte-Westfalia en Berlín, Alemania-, diseñada por Karl-Heinz Petzinka y Thomas Pink en el año 2002. El edificio se entiende a partir de la relación propuesta entre las pieles que conforman el sistema de envolvente (FIGS.07 A 10). La piel interior consiste en ventanas operables de piso a cielo (DVH), con persianas venecianas exteriores para el control solar y lumínico del espacio interior. La piel exterior se compone de láminas de vidrio simple, fijas a una estructura secundaria formada por elementos curvos de madera laminada. La geometría de las pieles genera espacios de transición contenidos dentro del sistema. Desde el exterior, el edificio se muestra como un prisma cuadrangular transparente -debido a la forma regular que asume la fachada-, sin embargo, la piel interior presenta quiebres que permiten la generación de un atrio de acceso y uno central de mayores dimensiones, con una apertura hacia la zona posterior del inmueble. Así, la generación del sistema de envolvente y, por consiguiente, de la forma del edificio, surge de la definición de los espacios de transición, comprendidos a su vez como parte de una estrategia global de mediación con el entorno.

El proyecto obliga también a concebir el edificio de oficinas bajo parámetros de eficiencia energética, a partir de los requerimientos ambientales del trabajador, con el fin de potenciar su rendimiento y el desempeño de las tareas laborales en un entorno confortable. No obstante, es necesario un cambio en la forma de diseñar edificios con estas características, dada la importancia relativa de la envolvente y sus relaciones con el espacio interior, determinadas por la utilización de espacios de transición.

De todas maneras, el diseño de la envolvente en forma paralela al desarrollo del proyecto de arquitectura no conduce a resultados adecuados, por lo que se deberá tender a una mayor sinergi a entre arquitectos, especialistas e industria, con el fin de impulsar proyectos integrados desde las primeras etapas de diseño, bajo un concepto global de mediación con el contexto climático. + 

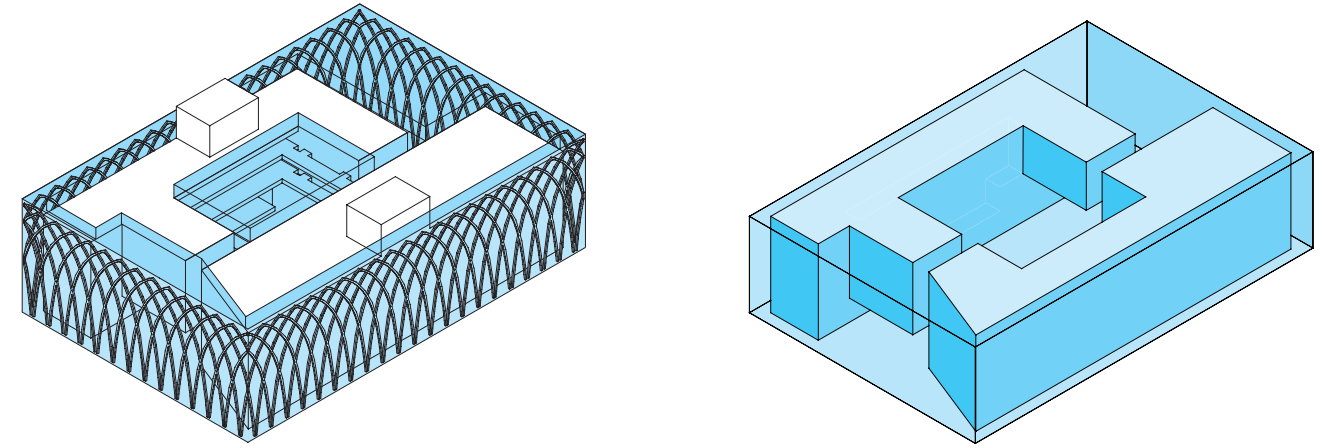

FIG.08 >

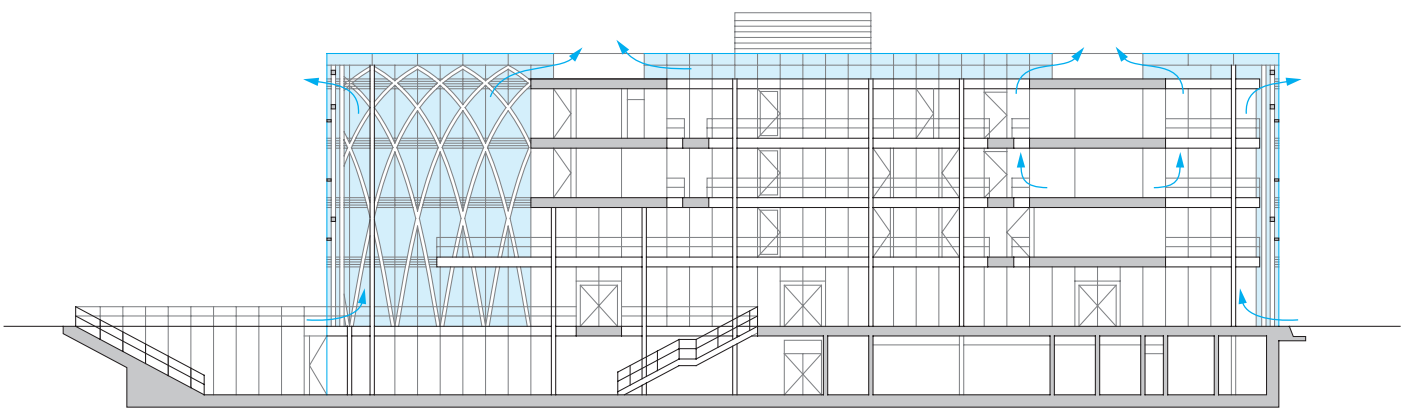

FIG.09 >

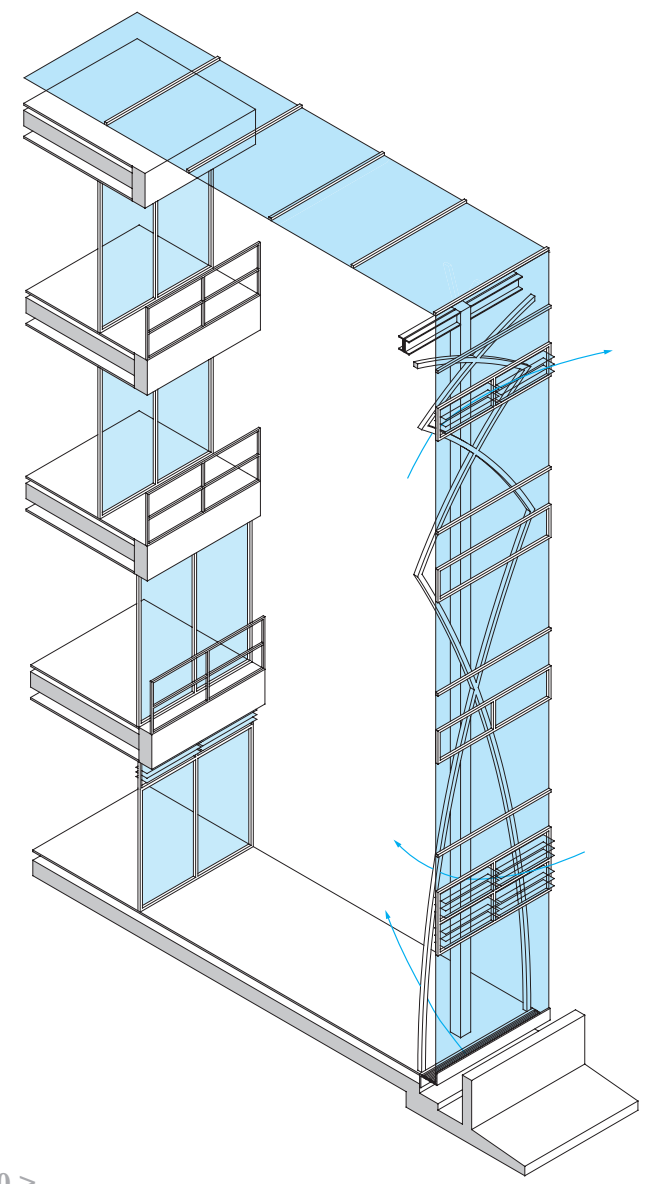

FIG.07 Embajada Renania. Petzinka + Pink, Berlín, 2002 Fuente: fotografía del autor.

FIG.08 Espacios de transición presentes en el edificio Fuente: elaboración propia.

FIG.09 Corte longitudinal.

Relación entre los distintos espacios de transición y la posibilidad de ventilación de las oficinas interiores. Fuente: elaboración propia,

FIG.10 Detalle de pieles que componen el sistema de envolvente. Fuente: elaboración propia.

LEYENDA

1. Celosías vidriadas para salida de aire

2. Capa exterior vidrio simple

3. Entrada/salida de aire

4. Estructura madera laminada

5. Montante de acero

6. Capa interior ventana corredera dvh

7. Toma de aire exterior

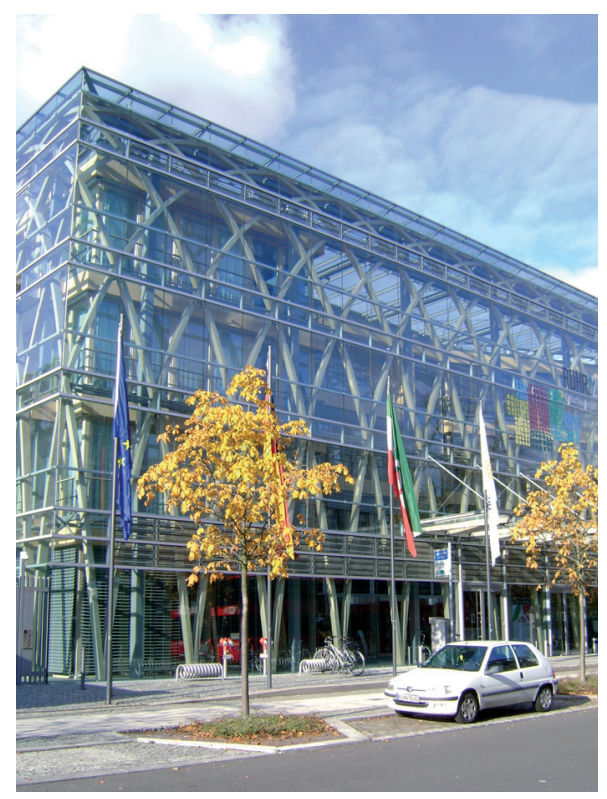

FIG.07 >

\section{Bibliografía sugerida}

ÁBALos, Iñaki. Técnica y arquitectura en la ciudad contemporánea. 1950-2000. Editorial Nerea, Hondarribia, 2000. / FERNÁNDEZ-GALIANO, Luis. "La vida es oficina”. AV Monografías, $\mathrm{N}^{\circ}$ 103. Editorial Arquitectura Viva S.L., Barcelona, septiembre-octubre, 2003. I HARTKOPF, Volker. "Mayor design changes for the office of the future". Designing The Office of the Future: The Japanese Approach to Tomorrow's Workplace. Editorial Wiley, Nueva York, 1993. I HASCHER, Rainer, et ál. Office Buildings: A Design Manual. Editorial Birkhäuser, Berlín, 2002. / LOFTNESS, Vivian. "The interior environment". En: GRECH, Chris y David walters. Future Office: Design, Practice and Applied Research. Editorial Routledge, Londres, 2008. I myerson, Jeremy. The Creative Office. Editorial Laurence King, Londres, 1999. / RUSSELL, James. "Arquitectura e ideales corporativos". AV Monografías, $\mathrm{N}^{\circ}$ 103. Editorial Arquitectura Viva S.L., Barcelona, septiembre-octubre, 2003. / VISCHER, Jacqueline. Space Meets Status: Designing Workplace Performance. Editorial Routledge, Londres, 2005. I "Towards a Psychology of the Work Environment". GRECH, Chris y David walters. Future Office: Design, Practice and Applied Research. Editorial Routledge, Londres, 2008. 